\title{
Modeling and Simulation of Biochemical Processes Using Stochastic Hybrid Systems: The Sugar Cataract Development Process
}

\author{
Derek Riley $^{1}$, Xenofon Koutsoukos ${ }^{1}$, and Kasandra Riley ${ }^{2}$ \\ ISIS/EECS \\ ${ }^{1}$ Vanderbilt University \\ Nashville, TN 37235, USA \\ Derek.Riley, Xenofon.Koutsoukos@vanderbilt.edu \\ Howard Hughes Medical Institute \\ ${ }^{2}$ Yale University \\ New Haven, CT, USA \\ Kasandra.Riley@yale.edu
}

\begin{abstract}
As biomedical research advances there is an increasing need to model and simulate more complicated systems to better understand them. Since biochemical processes are inherently stochastic and often contain both continuous and discrete behavior, stochastic hybrid systems are an ideal modeling paradigm for capturing their dynamics. In this paper we present a framework for modeling biochemical systems and demonstrate the approach for the sugar cataract development process including two methods of modeling drug treatment. Further, we present a simulation method that uses second-order Taylor approximations for the continuous dynamics and an improved method for detecting boundary hits. We use the sugar cataract development process to demonstrate the results of the method.
\end{abstract}

\section{Introduction}

As biomedical research advances into more complicated systems, there is an increasing need to model and simulate these systems to better understand them. Since biochemical processes are inherently stochastic and often contain both continuous and discrete behavior, Stochastic Hybrid Systems (SHS) are an ideal modeling paradigm for capturing their complex dynamics. Such systems are often too large and complex for exhaustive verification techniques, so accurate, efficient simulation techniques are very important.

Recently, a renewed interest in the field of biochemical system modeling has increased the quality and diversity of the models created. Biological protein regulatory networks have been modeled with hybrid systems using linear differential equations to describe the changes in protein concentrations and discrete switches to activate or deactivate the continuous dynamics based on protein thresholds [1. Biomolecular network modeling using hybrid systems is accomplished by using differential equations to model feedback mechanisms and discrete switches to 
model changes in the underlying dynamics [2]. A modeling technique that uses polynomial SHS to construct models for chemical reactions is presented in [3]. A SHS model of a genetic regulatory network is compared to a deterministic model in 4. Switching thresholds for piecewise-affine models of genetic regulatory networks are studied in [5]. SHS models of biochemical systems using reaction rate analysis have been developed and simulated in 6. A biochemical system drug model based on physical interactions at the molecular level has been developed in 7 .

Sugar Cataract Development (SCD) has been studied previously because it is a biomedically significant process, and the dynamics of the system are complex and difficult to test experimentally [8]. After a brief description of the SCD model presented in [6] and establishment of realistic parameters for its simulation, we present two new models of medication-controlled SCD. The first model incorporates the medication and models the effect that the medication has on the system. The second new model adds probabilistic delays to capture both the absorption of the drug into the system and drug metabolism.

Simulation of SHS is challenging because it must combine numerical integration methods for Stochastic Differential Equations (SDEs) and methods for detection of boundary crossings. Numerical integration of SDEs is accurate if the trajectory is sufficiently far from any boundaries; however, when the trajectory is close to a boundary, large errors can be incurred. A technique for accurately detecting absorbing boundaries has been developed for one-dimensional systems [9], and extensions have been proposed that scale to higher dimensional systems [10. The boundary crossing detection algorithm presented in [11] uses analysis of moments to improve the accuracy of the approximation.

To improve upon previous SHS simulation techniques we present the Hybrid Milstein Method (HMM) which utilizes a second order Taylor-based approximation for the stochastic continuous dynamics in conjunction with a technique for accurately approximating the boundary hitting times. We validate the SCD models by comparing simulation trajectories of the SHS and the stochastic simulation algorithm (SSA), which is considered to be an accurate but computationally inefficient approximation.

The organization for the rest of the paper is as follows: Section 2 describes the biochemical modeling framework as well as three SCD models, Section 3 describes the SHS simulation technique, Section 4 presents our simulation results, and Section 5 concludes the work.

\section{Modeling Biochemical Reactions Using SHS}

\subsection{Dynamics of Biochemical Reactions}

Discrete models are a natural modeling paradigm for biochemical systems because they can capture the changes of the concentrations of the involved reactants and products based on the stoichiometry defined by the biochemical reaction. In a discrete model, when the reaction fires, the concentrations of the reactants and products are reset to the appropriate updated values. 
To accurately model the reactions, the rate at which the individual reactions fire must be calculated. The rate at which chemical reactions occur is calculated using the stoichiometry defined by the type of reaction assuming temperature and pressure are constant. For example, the reaction $V+X \rightarrow Y+Z$, has a reaction rate $a=k v x$ where chemical species $V, X, Y$, and $Z$ have concentrations $v, x, y$, and $z$, and $k$ is the reaction's kinetic coefficient. The rates of other types of reactions can be calculated similarly [3].

Chemical reactions are inherently probabilistic because of the unpredictability of molecular motion 12. Discrete stochastic models of reactions describe a reaction as firing at a rate which is calculated using the chemical concentrations and the kinetic coefficient. Slow reactions occur when reaction rates and concentrations are small enough and they can be modeled and simulated efficiently using discrete stochastic techniques. However, discrete simulations become inefficient when there are large concentrations of molecules and/or fast reaction rates. When discrete models become inefficient, reactions can be accurately modeled as continuous stochastic models [6].

The rate of change of each chemical species in a fast reaction is calculated using the chemical dynamics from the biochemical reactions. Suppose that we have a system of $M$ chemical reactions and $N$ chemical species. We define $x_{i}$ as the concentration of the $i$ th chemical species in micro-Molarity $(\mu \mathrm{M}), M_{\text {fast }}$ as the number of fast reactions, $a_{j}$ as the reaction rate of the $j$ th reaction, $w$ as an $M_{\text {fast }}$-dimensional Wiener process, and the stoichiometric matrix $v$ as a $\left(M_{\text {fast }} \times N\right)$ matrix whose values represent the concentration of chemical species lost or gained in each reaction. The dynamics for each of the $i$ chemical species are described by

$$
d x_{i}=\sum_{j=1}^{M_{\text {fast }}} v_{j i} a_{j}(x(t)) d t+\sum_{j=1}^{M_{\text {fast }}} v_{j i} \sqrt{a_{j}(x(t))} d w_{j} .
$$

Biochemical systems can contain a mixture of both fast and slow reactions. When fast and slow dynamics must both be considered it is most efficient to use a combined, hybrid modeling approach to take advantage of the efficiency of continuous modeling for the fast reactions while still keeping the accuracy of discrete modeling for the slow reactions. Determining which reactions are fast or slow is based on analysis of the rates using the kinetic coefficients and chemical concentrations. To determine the slowest rate, the smallest possible concentrations for each chemical species are used. Similarly, the fastest rate can be determined by using the highest possible concentrations. Since the reaction rates depend on the concentrations, reactions may be classified as either fast or slow dynamically based on the system state.

\section{$2.2 \quad$ Medication Modeling}

Understanding how a biochemical system will operate under normal conditions is important; however, in many systems, it is advantageous to understand how the 
system will act when it is perturbed by outside influences such as a medication. The interaction of a drug with a biochemical system is important to model and analyze because often the anticipated affect of the drug is altered by unforeseen influences, and theoretical modeling and testing can help to demonstrate the safety of a medication before it is tested on real subjects.

Drugs are administered to patients to improve their health by altering the equilibrium of the reactions responsible for their symptoms. There are several defining characteristics of drugs that are considered when modeling their behavior. Drugs can generally be classified as either stimulants or inhibitors, which respectively increase or decrease reaction rates. The efficacy of a drug is the potential therapeutic response that it could produce. Drugs are metabolized by the body at varying rates further complicating the system, so the decay of the drug must be understood to accurately model its behavior.

The most direct drug modeling approach is to add the drug's chemical species and reactions to the original model. While this may appear to be a logical approach, it adds significant complexity to the system. A simpler technique is to model the behavior of a drug as an inhibitor or stimulant and avoid increasing the number of chemical reactions or chemical species considered.

Because stimulants and inhibitors alter the reaction rates of certain reactions, modeling the effect of a drug on a given chemical reaction can be accomplished by altering the kinetic coefficients. The amount of change of the kinetic coefficients is determined by the efficacy and metabolism rate of the drug. For the SCD model, discrete modes describe the system under different drug influences, and discrete transitions model the application and metabolism of the drug.

\subsection{Stochastic Hybrid Systems}

We adopt the model presented in 13. To establish the notation, we let $Q$ be a set of discrete states. For each $q \in Q$, we consider the Euclidean space $\mathbb{R}^{d(q)}$ with dimension $d(q)$ and we define an invariant as an open set $X^{q} \subseteq \mathbb{R}^{d(q)}$. The hybrid state space is denoted as $S=\bigcup_{q \in Q}\{q\} \times X^{q}$. Let $\bar{S}=S \cup \partial S$ and $\partial S=\bigcup_{q \in Q}\{q\} \times \partial X^{q}$ denote the completion and the boundary of $S$ respectively. The Borel $\sigma$-field in $S$ is denoted as $\mathcal{B}(S)$.

To define the execution of the system, we denote $(\Omega, \mathcal{F}, P)$ the underlying probability space, and consider an $\mathbb{R}^{p}$-valued Wiener process $w(t)$ and a sequence of stopping times $\left\{t_{0}=0, t_{1}, t_{2}, \ldots\right\}$. Let the state at time $t_{i}$ be $s\left(t_{i}\right)=\left(q\left(t_{i}\right), x\left(t_{i}\right)\right)$ with $x\left(t_{i}\right) \in X^{q\left(t_{i}\right)}$. While the continuous state stays in $X^{q\left(t_{i}\right)}, x(t)$ evolves according to the SDE

$$
d x=b(q, x) d t+\sigma(q, x) d w
$$

where the discrete state $q(t)=q\left(t_{i}\right)$ remains constant. A sample path of the stochastic process is denoted by $x_{t}(\omega), t>t_{i}, \omega \in \Omega$.

The next stopping time $t_{i+1}$ represents the time when the system transitions to a new discrete state. The discrete transition occurs either because the continuous 
state $x$ exits the invariant $X^{q\left(t_{i}\right)}$ of the discrete state $q\left(t_{i}\right)$ (guarded transition) or based on an exponential distribution with nonnegative transition rate function $\lambda: \bar{S} \rightarrow \mathbb{R}_{+}$(probabilistic transition). At time $t_{i+1}$ the system will transition to a new discrete state and the continuous state may jump according to the transition measure $R: \bar{S} \times \mathcal{B}(\bar{S}) \rightarrow[0,1]$. The evolution of the system is then governed by the SDE (2) with $q(t)=q\left(t_{i+1}\right)$ until the next stopping time. If $t_{i+1}=\infty$, the system continues to evolve according to (2) with $q(t)=q\left(t_{i}\right)$.

The following assumptions are imposed on the model. The functions $b(q, x)$ and $\sigma(q, x)$ are bounded and Lipschitz continuous in $x$ for every $q$, and thus the SDE (2) has a unique solution for every $q$. The transition rate function $\lambda$ is a bounded and measurable function which is assumed to be integrable for every $x_{t}(\omega)$. For the transition measure, it is assumed that $R(\cdot, A)$ is measurable for all $A \in \mathcal{B}(S)$ and $R(s, \cdot)$ is a probability measure for all $s \in \bar{S}$, and $R((q, x), d z)$ is a stochastic continuous kernel. Let $N_{t}=\sum_{i} I_{t \geq t_{i}}$ denote the number of jumps in the interval $[0, t]$. It is assumed that the expected number of jumps is finite for every initial state $s \in S$, that is $E_{s}\left[N_{t}\right]<\infty$. A sufficient condition for ensuring finitely many jumps can be formulated by restricting $R(s, A)$ [14 15].

\subsection{Sugar Cataract Modeling}

This section describes three SHS models of the biochemical process of SCD. The first model describes the biochemical process of SCD. The two subsequent models extend the first model to include the effect of medication on the system. The first medicated model assumes that the effect of the drug on the system is instantaneous, while the final model is designed to incorporate probabilistic delay to model absorption and metabolization.

Sugar Cataract Development Model (SCD1). A sugar cataract distorts the light passing through the lens of an eye by attracting water to the lens when an excess of sorbitol is present. Often these cataracts are formed in the eyes of diabetic patients who have highly fluctuating blood sugar levels. Several factors affect the accumulation of sorbitol including the amount of the enzyme SDH. SDH catalyzes the reversible oxidation of sorbitol and other polyalcohols to the corresponding keto-sugars 8 . There are 8 chemical species involved in the reaction: $N A D H\left(x_{1}\right), E-N A D H\left(x_{2}\right), N A D^{+}\left(x_{3}\right), E-N A D^{+}\left(x_{4}\right), \mathrm{SDH}$ $\left(x_{5}\right)$, Fructose $\left(x_{6}\right)$, Sorbitol $\left(x_{7}\right)$, and the inactive form of SDH $(Z)$.

A SHS model for SCD (SCD1) has been previously presented in 6 16. The ranges are bounded and are estimated using realistic concentration values derived from experimental data and Michaelis-Menten constants $(\mathrm{Km})$ defined as the rate of the reaction at half-maximal velocity [8]. Table 1 describes the seven reactions and rates involved in SCD. The rates are calculated based on the concentrations and the kinetic coefficients presented in Table 1.

Each of the six fast reactions are modeled using the SDE (1). The inactive form of $\mathrm{SDH}(\mathrm{Z})$ is not a reactant in any of the chemical equations, so its concentration is not modeled. The equations describe the rates of change of the individual chemical species and are 
Table 1. Sugar cataract reactions and kinetic coefficients

\begin{tabular}{|c|c|c|}
\hline Reaction & Kinetic coefficient & Rate \\
\hline$S D H+N A D H \rightarrow E-N A D H$ & $k_{1}=6.2$ & 31.1 \\
$E-N A D H \rightarrow S D H+N A D H$ & $k_{2}=33$ & 151 \\
$E-N A D H+F \rightarrow E-N A D^{+}+S$ & $k_{3}=0.0022$ & 6 \\
$E-N A D^{+}+S \rightarrow E-N A D H+F$ & $k_{4}=0.0079$ & 19.5 \\
$E-N A D^{+} \rightarrow S D H+N A D^{+}$ & $k_{5}=227$ & 998 \\
$S D H+N A D^{+} \rightarrow E-N A D^{+}$ & $k_{6}=.61$ & 3.2 \\
$S D H \rightarrow Z$ & $k_{7}=0.0019$ & 0.002 \\
\hline
\end{tabular}

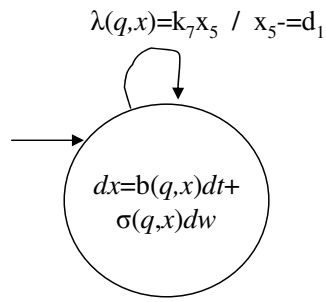

Fig. 1. SHS model of SCD1

$$
\begin{aligned}
d x_{1}= & \left(-k_{1} x_{1} x_{5}+k_{2} x_{2}\right) d t-\sqrt{k_{1} x_{1} x_{5}} d w_{1}+\sqrt{k_{2} x_{2}} d w_{2} \\
d x_{2}= & \left(k_{1} x_{1} x_{5}-k_{2} x_{2}-k_{3} x_{2} x_{6}+k_{4} x_{4} x_{7}\right) d t+\sqrt{k_{1} x_{1} x_{5}} d w_{1} \\
& -\sqrt{k_{2} x_{2}} d w_{2}-\sqrt{k_{3} x_{2} x_{6}} d w_{3}+\sqrt{k_{4} x_{4} x_{7}} d w_{4} \\
d x_{3}= & \left(k_{5} x_{4}-k_{6} x_{3} x_{5}\right) d t+\sqrt{k_{5} x_{4}} d w_{5}-\sqrt{k_{6} x_{3} x_{5}} d w_{6} \\
d x_{4}= & \left(k_{3} x_{2} x_{6}-k_{4} x_{4} x_{7}-k_{5} x_{4}+k_{6} x_{3} x_{5}\right) d t+\sqrt{k_{3} x_{2} x_{6}} d w_{3} \\
& -\sqrt{k_{4} x_{4} x_{7}} d w_{4}-\sqrt{k_{5} x_{4}} d w_{5}+\sqrt{k_{6} x_{3} x_{5}} d w_{6} \\
d x_{5}= & \left(-k_{1} x_{1} x_{5}+k_{2} x_{2}+k_{5} x_{4}-k_{6} x_{3} x_{5}\right) d t-\sqrt{k_{1} x_{1} x_{5}} d w_{1} \\
& +\sqrt{k_{2} x_{2}} d w_{2}+\sqrt{k_{5} x_{4}} d w_{5}-\sqrt{k_{6} x_{3} x_{5}} d w_{6} \\
d x_{6}= & \left(-k_{3} x_{2} x_{6}+k_{4} x_{4} x_{7}\right) d t-\sqrt{k_{3} x_{2} x_{6}} d w_{3}+\sqrt{k_{4} x_{4} x_{7}} d w_{4} \\
d x_{7}= & \left(k_{3} x_{2} x_{6}-k_{4} x_{4} x_{7}\right) d t+\sqrt{k_{3} x_{2} x_{6}} d w_{3}-\sqrt{k_{4} x_{4} x_{7}} d w_{4}
\end{aligned}
$$

The single slow reaction $S D H \rightarrow Z$ describes the conversion of the enzyme $(\mathrm{SDH})$ into its inactive form at a rate of $k_{7} x_{5}$. When the reaction occurs, the number of molecules of $x_{5}$ is decreased by one and the concentration is decreased by $d_{1}=10^{-21} \mu \mathrm{M}$. The SHS model can be seen in Figure 1. The reset on the transition $\left(x_{5}-=d_{1}\right)$ describes the effect of the single slow reaction on the concentration of $x_{5}$. For the SCD system, the classifications of the reactions do not change dynamically because the kinetic coefficients are significantly different and the chemical concentrations do not fluctuate widely. 
SCD Model with Medication Control (SCD2). Drugs can help patients who are at high risk of developing sugar cataracts. These drugs work by inhibiting the enzyme SDH thereby reducing the rate at which SDH reacts with other molecules in the system. This initially results in less sorbitol production; however, since the reversible reactions are tightly coupled, the results can have side effects such as increasing the fructose levels.

We have created a new SHS model (SCD2), shown in Figure 2 of drugmodulated SCD to include the effect that drug has on the system. The application of the drug is represented as a new discrete mode that represents drug-influenced dynamics where the reaction rates $k_{1}, k_{6}$, and $k_{7}$ are reduced by $50 \%$ to model the inhibition of the enzyme. Since the drug is metabolized slowly and the amount that the rates are reduced is directly proportional to the concentration of the drug, modeling a constant concentration is a reasonable approximation.

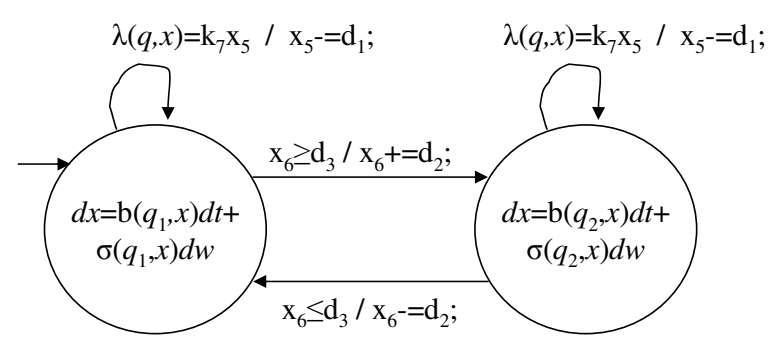

Fig. 2. SHS model of medication-controlled SCD2

We have modeled the drug administration based on an elevated level of fructose. It is assumed that patients self-monitor and self-administer the medication. When the amount of fructose in the blood rises above a threshold $d_{3}=250 \mu \mathrm{M}$, we use a guarded transition to drive the system to a new state which introduces the effect of the drug. When the fructose level drops back below $d_{3}$, we use another guarded transition to transition to the original state effectively removing the effect of the drug. We also include resets on the mode transitions to avoid infinitely fast switching that arises due to the stochastic nature of the Wiener process. The reset increases or decreases the fructose concentration by $d_{2}=1 \mu \mathrm{M}$.

SCD Model with Probabilistically-Delayed Medication Effect (SCD3). The SCD2 model is effective for demonstrating the effect of medication on the reactions; however, realistically the effect of the drug will not be immediate because of variable drug metabolism rates. Drugs are generally administered in a form called a prodrug which allows the transport of the actual drug to the appropriate cells. This prodrug is metabolized into an active form of the drug at different rates for different people. Furthermore, once a patient discontinues taking a drug, the body can metabolize the residual drug at variable rates depending on many factors. 


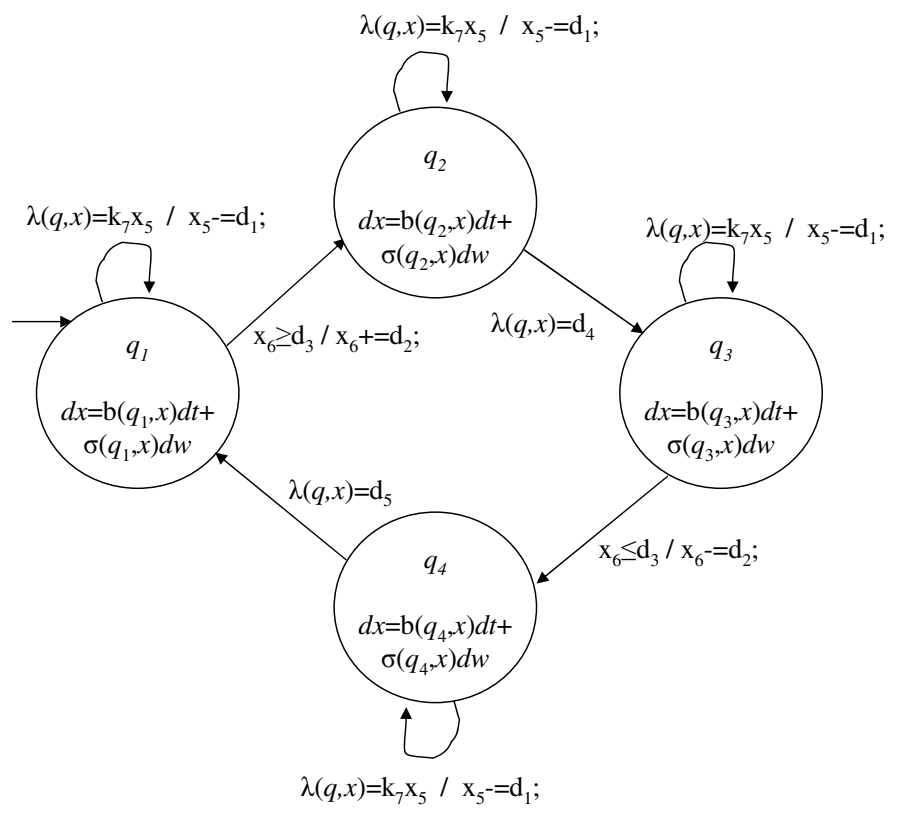

Fig. 3. SHS model of medication-controlled SCD3 with delays

We have developed a model (SCD3), seen in Figure 3, which incorporates two new states to model the delay of the conversion from prodrug to drug $\left(q_{2}\right)$ and metabolism after dosage is discontinued $\left(q_{4}\right)$. We use guarded transitions to model exiting the medicated and non-medicated states and entering the respective delay states. We then use probabilistic transitions to model the exit from the delay states to model the stochastic nature of the conversion and metabolism rates. The value $d_{4}=0.05$ is the rate of an exponential distribution that models the delay incurred by the conversion of prodrug to drug, and $d_{5}=0.05$ is the value which models the exponential distribution corresponding to the drug metabolism delay. These values were chosen so the average delay is on the order of one hour which is reasonable for the SCD system, but the values could be easily changed to model other types of medications. SHS can also incorporate the continuous state into the transition rate if such a model is necessary.

The continuous dynamics of the medicated $\left(q_{3}\right)$ and non-medicated $\left(q_{1}\right)$ states are consistent with SCD2. The dynamics of the delay state $q_{2}$ are the same as those in state $q_{1}$ to reflect the lack of change while the prodrug is being converted into the drug. The dynamics in the delay state $q_{4}$ model the metabolism of the drug after the administration is removed, so the kinetic coefficients are adjusted to reflect their half-life values. The coefficients can be adjusted to model various drugs. 


\section{SHS Simulation}

\subsection{Background}

Simulating SHS is an important task because it can be used to understand and validate models. However, it is challenging because the interplay between the stochastic continuous and discrete dynamics can cause large errors if handled incorrectly. Errors can be decreased by reducing the step size of the approximation, but this comes at the cost of efficiency. Therefore, care must be taken to ensure that the simulation techniques used are accurate and efficient.

For numerical integration of SDEs, the order of convergence is used to quantify the quality of the approximation. An approximation $X^{\Delta t}(T)$ at time $T$ with step size $\Delta t$ converges with order $\gamma$ strongly to the actual trajectory $x(T)$ if there exists $c>0$ such that $E\left(\left|x(T)-X^{\Delta t}(T)\right|\right) \leq c \Delta t^{\gamma} . X^{\Delta t}(T)$ converges with order $\gamma$ weakly to $x(T)$ if there exists $c>0$ such that $E\left(\left|f(x(T))-f\left(X^{\Delta t}(T)\right)\right|\right) \leq$ $c \Delta t^{\gamma}$ for a given class of measurable functions $f$ [17. Strong convergence implies that the trajectory is a possible trajectory of the system, and weak convergence implies that the computed trajectory only preserves the moments of the actual trajectory.

Simulation of SDEs can be performed using Taylor-based approximation techniques which have strong order of convergence of $\gamma=0.5$ to $\gamma=3.0$ and weak order of convergence of $\gamma=1.0$ to $\gamma=6.0$ depending on the number of approximating terms [17. The computation of higher order terms requires many more operations and can be prohibitively expensive; therefore, a tradeoff must be reached to achieve the appropriate accuracy and efficiency.

Numerical integration methods for SDEs assume that the solution is far away from any boundaries; however, this assumption does not hold for SHS where the effect of the switching boundaries must be taken into account. Large errors can be incurred if the boundary conditions are not handled properly. Let us assume a system has an invariant $X^{q}$ with a boundary $\partial X_{q}$, and the state at time $t$ is $X(t)$. As shown in Figure 4, if $\Delta t$ is large, it is possible that $X(t), X(t+\Delta t) \in X^{q}$, but $\exists \tau \in[t, t+\Delta t]$ where $x(\tau) \notin X^{q}$. In this case, a discrete transition will occur in the actual execution of the SHS but not in the approximating solution, and this discrepancy may cause a significant error.

\subsection{Simulation of SDEs}

Simulation of SDEs can be performed using the Euler Maruyama (EM) method which is a first-order Taylor scheme [17. Assuming a $d$-dimensional drift coefficient $b$ and a $d \times m$ diffusion coefficient $\sigma$, the $k$ th component of the EM scheme is given by

$$
X_{n+1}^{k}=X_{n}^{k}+b^{k} \Delta t+\sum_{j=1}^{m} \sigma^{k, j} \Delta W^{j}
$$

for $k=1,2, \ldots, d$ where $\Delta W^{j}$ is the normally-distributed increment of the $j$ th component of the $m$-dimensional Wiener process $W$. The EM method is simple 


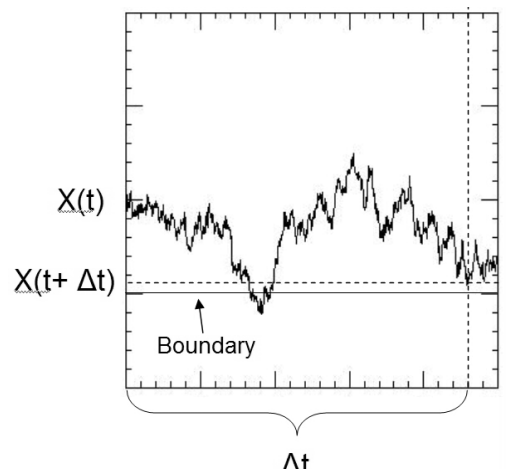

Fig. 4. A SHS trajectory close to a boundary

to implement, but achieves a strong convergence of $\gamma=0.5$ and weak convergence $\gamma=1.0$, so small time steps must be used to generate accurate approximations.

The Milstein Method (MM) is a second-order Taylor scheme and has a strong order of convergence $\gamma=1.0$ while maintaining an acceptable efficiency. The $k$ th component of the MM scheme is described by

$$
X_{n+1}^{k}=X_{n}^{k}+b^{k} \Delta t+\sum_{j=1}^{m} \sigma^{k, j} \Delta W^{j}+\sum_{j_{1}, j_{2}=1}^{m} L^{j_{1}} \sigma^{k, j_{2}} I_{\left(j_{1}, j_{2}\right)}
$$

where

$$
L^{j}=\sum_{k=1}^{d} \sigma^{k, j} \frac{d}{d x^{k}} \text { and } I_{\left(j_{1}, j_{2}\right)}=\int_{\tau_{n}}^{\tau_{n+1}} \int_{\tau_{n}}^{s_{1}} d w_{s_{2}}^{j_{1}} d w_{s}^{j_{2}} .
$$

A method for approximating the multiple stochastic integrals is given in 17 .

\subsection{Switching Boundaries}

Once the execution of the SHS hits a switching boundary, the current process is stopped (absorbed) and re-started in a new state; therefore, switching boundaries can be treated as absorbing boundaries. It is important to accurately estimate the time and location that the process is absorbed to minimize the error introduced into the approximation.

The naive technique to detect that a boundary was hit is to analyze the trajectory at each time step to determine if it has crossed the boundary or not. This method has a strong order of convergence of $\gamma=0.5$ [11]. The technique developed in 18 determines whether or not the trajectory has hit an absorbing boundary with weak order $\gamma=1.0$ assuming that the boundary is sufficiently smooth. We assume that the switching boundaries are hyperplanes $\partial X^{q}=\left\{x \in \mathbb{R}^{d(q)}: n .\left(x-X_{b}\right)=0\right\}$ where $n$ is the unit vector normal to the boundary $\partial X^{q}, X_{b}$ is the position of the boundary, and denote $X_{t}, X_{t+\Delta t}$ the 
computed continuous state at time $t$ and $t+\Delta t$ respectively. If $X_{t+\Delta t}$ hasn't crossed the boundary, but is close, the probability that the state trajectory has hit the boundary between $t$ and $t+\Delta t$ is

$$
P(h i t)=\exp \left(\frac{-2\left(n \cdot\left(X_{t}-X_{b}\right)\right)\left(n \cdot\left(X_{t+\Delta t}-X_{b}\right)\right)}{n \cdot\left(\sigma \sigma^{*}\left(X_{t}\right) n\right) \Delta t}\right) .
$$

For this approximation to be accurate, $\sigma \sigma^{T}\left(X_{t}\right)$ must be non-degenerate in the direction normal to the boundary [18. In between steps of the MM, the probability $P($ hit $)$ is tested against a uniform random value $U$ in $[0,1]$, and when $P(h i t)<U$, we assume that the absorbing boundary has been hit.

Firing of the probabilistic transitions (according to the transition rate $\lambda$ ) occurs according the technique described in [15. We draw a sample from a uniform distribution and test the exponential decay at various times to determine the jump time for each probabilistic transition. When the exponential decay is greater than or equal to the random value, the transition is fired.

We combine the absorbing boundary version of the MM and probabilistic firing technique to create the Hybrid Milstein Method (HMM) simulation technique for SHS. The resulting algorithm has a weak convergence of $\gamma=1.0$. The following algorithm describes a version of one step of the simulation method.

\section{Algorithm 3.1: HighOrderSHSSimStep $\left(X_{t}^{k}\right.$, SimLength $)$}

$$
\begin{aligned}
& X_{t+\Delta t}^{k}=X_{t}^{k}+b^{k} \Delta t+\sum_{j=1}^{m} \sigma^{k, j} \Delta W^{j}+\sum_{j_{1}, j_{2}=1}^{m} L^{j_{1}} \sigma^{k, j_{2}} I_{\left(j_{1}, j_{2}\right)} \\
& t++ \\
& \text { if } U 1=\operatorname{rand}(0,1)<\exp \left(\frac{-2\left(n \cdot\left(X_{t}^{k}-X_{b}\right)\right)\left(n \cdot\left(X_{t+\Delta t}^{k}-X_{b}\right)\right)}{n \cdot\left(\sigma \sigma^{*}\left(X_{t}\right) n\right) \Delta t}\right) \\
& \text { then FireGuardedTransition } \\
& \text { if } U 2=\operatorname{rand}(0,1)<\exp (-\lambda(t-\text { TimeOfLastFire })) \\
& \text { then FireProbabilisticTransition }
\end{aligned}
$$

\section{Simulation Results}

To better understand and validate our models, we present simulation results using variants of the SSA, EM, and MM methods. The SSA simulates chemical reactions consuming reactants and creating products one reaction at a time. Individual reactions in a system are assigned probabilities of occurrence, and probability distributions are used to choose which reaction fires at each iteration. Once a reaction fires, the quantities of reactants and products are updated [19. The SSA is very accurate, but it can be inefficient for large systems or fast reactions because many iterations must be completed before results can be observed. To efficiently handle practical systems, computational improvements such as R-leaping have been devised for the SSA [20. R-leaping increases the number of reactants consumed and products produced in each step by a factor of R. This increases the efficiency of the approximation, but will decrease the accuracy. Since updates are made based on concentrations, the overall time step can vary throughout the simulation. 

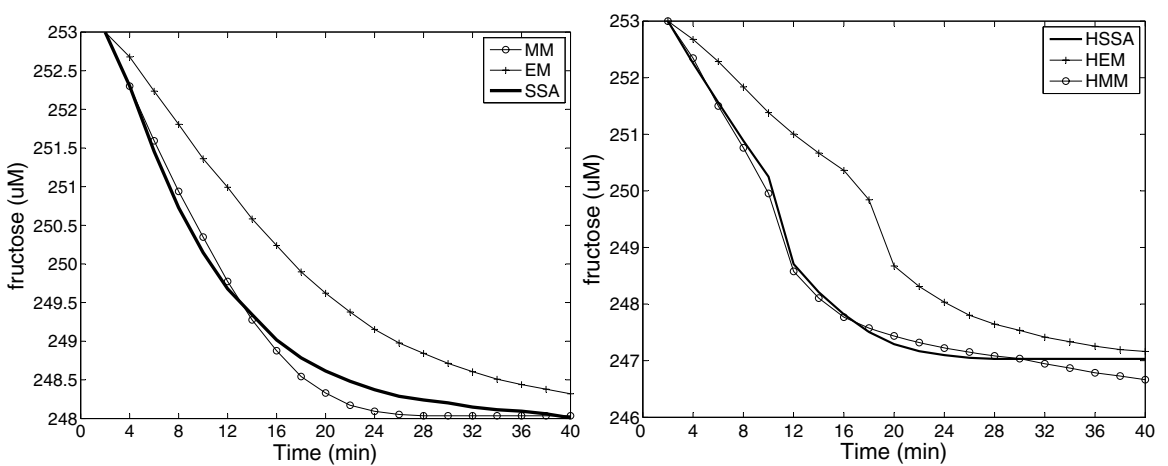

Fig. 5. Simulation results for SCD1 and SCD2
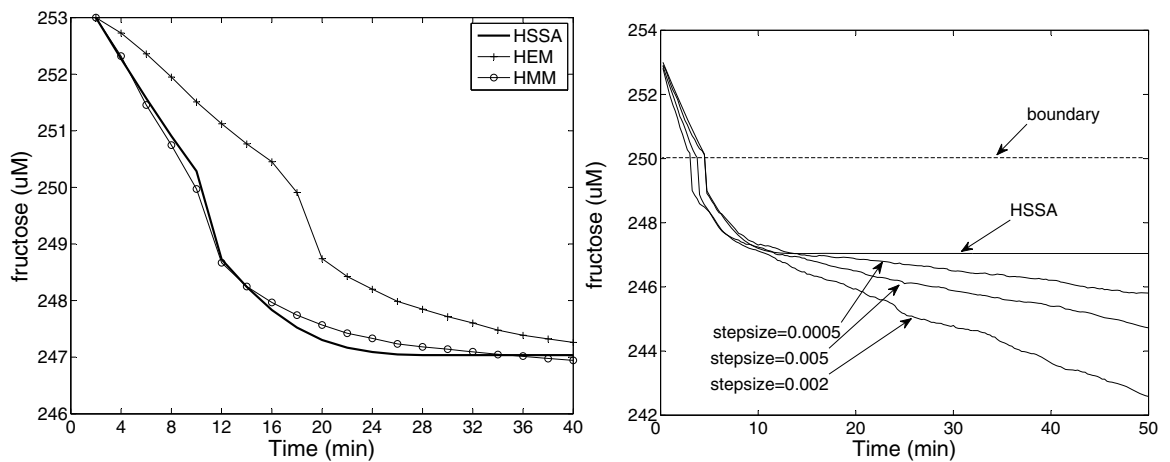

Fig. 6. Simulation results for SCD3 and stepsize comparison

For simulation of SCD2 and SCD3, we have created a new algorithm, the Hybrid Stochastic Simulation Algorithm (HSSA), which implements the SSA using R-leaping and discrete transitions between modes. After each iteration of the SSA, the guards for all valid transitions are tested, and a transition which validates its guard conditions is fired if possible. Once the transition resets have been executed, the SSA algorithm resumes in the new state.

The stochastic continuous dynamics of the SCD models can be simulated using EM approximations 17. To accurately model the discrete transitions of the SCD models, we have developed a variant of the EM approximations called the Hybrid EM (HEM). In HEM, discrete transitions are incorporated into the EM approximations by analyzing transition guards between steps of the continuous dynamics simulation and executing the resets when a boundary crossing is detected. Once the state is updated, the EM algorithm continues in the new discrete state. We use the $H M M$ as described in the previous section to provide a more accurate simulation result.

Since the SSA algorithm is considered to be an accurate approximation of a well-stirred chemical system [20], we compare the results of our HSSA, HEM, 
and $H M M$ algorithms to demonstrate accuracy of the three SHS models and the accuracy of the approximations in Figures 5 and 6. We also present a comparison of the accuracy of the HMM for SCD3 at various resolutions in Figure 6. We chose to display the concentration of fructose because it is directly correlated with the development of cataracts, and it affects the administration of the medication. The initial conditions of the system are: $x_{1}=5.0, x_{2}=0.0, x_{3}=5.0$, $x_{4}=0.0, x_{5}=1.0, x_{6}=253.0$, and $x_{7}=0$. The figures display the average concentration at each time step for fructose for 100 runs of the three models. These results show that the high order simulation technique results in a more accurate simulation with a larger step size resulting in a faster simulation. The 100 HSSA simulations completed in 98 hours, the 100 HEM simulations took approximately 6 hours (with step size $\Delta t=0.00001$ ), and the $H M M$ simulations took approximately 3 hours (with step size $\Delta t=0.0005$, and $p=1$ 迆) on a $3 \mathrm{GHz}$ desktop computer.

\section{Conclusions and Future Work}

Accurate and efficient simulation of SHS is an important task because it is an important tool which can expose the intricacies of the complicated dynamics of highly-coupled systems. The interplay between the continuous and discrete dynamics in SHS can introduce large errors into the simulations, so they must be handled carefully. Our technique using high-order methods for simulating SDEs combined with probabilistic boundary detection improves the accuracy and efficiency of the simulator when compared with the naive approaches. This work only addresses absorbing boundaries, so in the future we will also incorporate boundary conditions for reflecting boundaries that are required for practical systems such as biochemical processes.

Acknowledgements. This research is partially supported by the National Science Foundation (NSF) CAREER grant CNS-0347440.

\section{References}

1. Ghosh, R., Tomlin, C.: Symbolic reachable set computation of piecewise affine hybrid automata and its application to biological modeling: Delta-notch protein signalling. Sys. Bio. 1, 170-183 (2004)

2. Alur, R., Belta, C., Ivanicic, F., Kumar, V., Mintz, M., Pappas, G., Rubin, H., Schug, J.: Hybrid modeling and simulation of biomolecular networks. In: Di Benedetto, M.D., Sangiovanni-Vincentelli, A.L. (eds.) HSCC 2001. LNCS, vol. 2034, pp. 19-33. Springer, Heidelberg (2001)

3. Hespanha, J., Singh, A.: Stochastic models for chemically reacting systems using polynomial stochastic hybrid systems. Int. J. on Robust Cont., Special Issue on Control at Small Scales 15, 669-689 (2005)

\footnotetext{
${ }^{1} \mathrm{p}$ is a parameter used to approximate the Stratonovich integrals as described in [17.
} 
4. $\mathrm{Hu}$, J., Wu, W., Sastry, S.: Modeling subtilin production in bacillus subtilis using stochastic hybrid systems. In: Alur, R., Pappas, G.J. (eds.) HSCC 2004. LNCS, vol. 2993, pp. 417-431. Springer, Heidelberg (2004)

5. Drulhe, S., Ferrari-Trecate, G., de Jong, H., Viari, A.: Reconstruction of switching thresholds in piecewise-affine models of genetic regulatory networks. In: Hespanha, J.P., Tiwari, A. (eds.) HSCC 2006. LNCS, vol. 3927, pp. 184-199. Springer, Heidelberg (2006)

6. Salis, H., Kaznessis, Y.: Accurate hybrid stochastic simulation of a system of coupled chemical or biochemical reactions. J. Chem. Phys. 122, 54-103 (2005)

7. Ramos, M., Melo, A., Henriques, E., Gomes, J., Reuter, N., Maigret, B., Floriano, W., Nascimento, M.: Modeling enzyme-inhibitor interactions in serine proteases. Int. J. Quant. Chem. 74(3), 299-314 (1999)

8. Marini, I., Bucchioni, L., Borella, P., Corso, A.D., Mura, U.: Sorbitol dehydrogenase from bovine lens: Purification and properties. Arch. Biochem. and Biophy. 340, 383-391 (1997)

9. Mannella, R.: Absorbing boundaries and optimal stopping in a stochastic differential equation. Phys. Lett. A 254, 257-262 (1999)

10. Lamm, G.: Extended brownian dynamics. iii. three dimensional diffusion. J. Chem. Phys. 80(6), 2845-2855 (1983)

11. Peters, E., Barenbrug, T.: Efficient brownian dynamics simulation of particles near walls. i. reflecting and absorbing walls. Physical Review 66, 1-7 (2002)

12. Elowitz, M., Levine, A., Siggia, E., Swain, P.: Stochastic gene expression in a single cell. Science 1183(297) (2002)

13. Bujorianu, M., Lygeros, J.: Theoretical foundations of general stochastic hybrid systems: Modeling and optimal control. In: IEEE Conf. on Dec. and Cont. (2004)

14. Koutsoukos, X., Riley, D.: Computational methods for reachability analysis of stochastic hybrid systems. In: Hespanha, J.P., Tiwari, A. (eds.) HSCC 2006. LNCS, vol. 3927, pp. 377-391. Springer, Heidelberg (2006)

15. Bernadskiy, M., Sharykin, R., Alur, R.: Structured modeling of concurrent stochastic hybrid systems. In: Lakhnech, Y., Yovine, S. (eds.) FORMATS 2004 and FTRTFT 2004. LNCS, vol. 3253, pp. 309-324. Springer, Heidelberg (2004)

16. Riley, D., Koutsoukos, X., Riley, K.: Safety analysis of sugar cataract development using stochastic hybrid systems. In: Bemporad, A., Bicchi, A., Buttazzo, G. (eds.) HSCC 2007. LNCS, vol. 4416, pp. 758-761. Springer, Heidelberg (2007)

17. Kloeden, P., Platen, E.: Numerical Solution of Stochastic Differential Equations. Springer, Heidelberg (1999)

18. Gobet, E.: Euler schemes and half-space approximation for the simulation of diffusion in a domain. ESAIM: Probability and Statistics 5, 261-297 (2001)

19. Gillespie, D.: A general method for numerically simulating the stochastic time evolution of coupled chemical reactions. J. Comp. Phys. 22, 403-434 (1976)

20. Auger, A., Chatelain, P., Koumoutsakos, P.: R-leaping: Accelerating the stochastic simulation algorithm by reaction leaps. J. Chem. Phys. 125, 84-103 (2006) 